\title{
Immobilization of Prussian blue nanoparticles in acrylic acid-surface functionalized poly(vinyl alcohol) sponges for cesium adsorption
}

\author{
Hyobin $\mathrm{Wi}^{1}$, Sung-Won Kang ${ }^{2}$, Yuhoon Hwang ${ }^{{ }^{+}}$ \\ ${ }^{1}$ Department of Environmental Engineering, Seoul National University of Science and Technology, Seoul 01811, Republic of Korea \\ ${ }^{2}$ Environmental and Plant Engineering Research Institute, Korea Institute of Civil Engineering and Building Technology, Goyang 10223, Republic of Korea
}

\begin{abstract}
Prussian blue (PB) is known to be an effective material for radioactive cesium adsorption, but its nano-range size make it difficult to be applied for contaminated water remediation. In this study, a simple and versatile approach to immobilize PB in the supporting matrix via surface functionalization was investigated. The commercially available poly vinyl alcohol (PVA) sponge was functionalized by acrylic acid (AA) to change its major functional group from hydroxyl to carboxylic, which provides a stronger ionic bond with PB. The amount of AA added was optimized by evaluating the weight change rate and iron(III) ion adsorption test. The FTIR results revealed the surface functional group changing to a carboxyl group. The surface functionalization enhanced the attachment of $\mathrm{PB}$, which minimized the leaching out of $\mathrm{PB}$. The $\mathrm{Cs}^{+}$adsorption capacity significantly increased due to surface functionalization from 1.762 to $5.675 \mathrm{mg} / \mathrm{g}$. These findings showed the excellent potential of the PB-PAA-PVA sponge as a cesium adsorbent as well as a versatile approach for various supporting materials containing the hydroxyl functional group.
\end{abstract}

Keywords: Acrylic acid, Cesium adsorbent, Poly vinyl alcohol sponge, Prussian blue, Radioactive material, Surface functionalization

\section{Introduction}

${ }^{137} \mathrm{Cs}$, which is relatively light compared with other radioactive materials, became a more hazardous substance when the nuclear power plant accident happened in Fukushima, Japan. Their lightweight property makes cesium(I) likely to be dispersed in aqueous conditions or the atmosphere, and hydrated cesium ion can be found in many areas in Japan and other countries [1, 2]. Because of the radioactive characteristics of ${ }^{137} \mathrm{Cs}$, the most proper way to decontaminate is to use an adsorbent to concentrate it and keep in a separated area. Therefore, many types of adsorbents have been suggested for cesium removal.

Metal hexacyanoferrates(II), including Prussian blue (PB), has a unique structure to capture cesium(I) ion effectively. They have special lattice structure having room to capture hydrated cesium ions by electrostatic forces as well as size exclusion. It means that two adsorption property of chemisorption and phsysorption can be applied together [3].
Specifically, $\mathrm{PB}, \mathrm{Fe}^{(\mathrm{III})}{ }_{4}\left[\mathrm{Fe}^{(\mathrm{II})}(\mathrm{CN})_{6}\right]_{3}$, has a property capturing cation via ion exchange [4]:

$$
\begin{aligned}
& \mathrm{Fe}_{4}^{I I I}\left[\mathrm{Fe}^{I I}(\mathrm{CN})_{6}\right]_{3}+4 e^{-}+4 K^{+} \rightarrow \\
& K_{4} \mathrm{Fe}_{4}^{I I}\left[\mathrm{Fe}^{I I}(\mathrm{CN})_{6}\right]_{3}+4 \mathrm{Cs}^{+} \rightarrow \\
& \mathrm{Cs}_{4} \mathrm{Fe}_{4}^{I I}\left[\mathrm{Fe}^{I I}(\mathrm{CN})_{6}\right]_{3}+4 K^{+}
\end{aligned}
$$

The affinity of $\mathrm{PB}$ towards various cation was reported as $\mathrm{Cs}^{+}>\mathrm{Ca}^{+}>\mathrm{K}^{+}>\mathrm{NH}_{4}{ }^{+}>\mathrm{Na}^{+}>\mathrm{Mg}^{+}[5]$.

However, the size of the PB particle is $5-200 \mathrm{~nm}$ size and it can be readily dispersed in water, which makes it difficult to recover it after adsorption takes place. The composite materials containing PB in/on the supporting matrix have been reported as being able to enhance the applicability of $\mathrm{PB}$ in environmental remediation [6-9]. There are several criteria to be satisfied in order to be considered good PB composite materials such as high cesium adsorption capacity, low PB leaching in water, and

Received May 21, 2018 Accepted July 12, 2018

${ }^{\dagger}$ Corresponding author

Email: yhhwang@seoultech.ac.kr

Tel: +82-2-970-6626 Fax: +82-2-971-5776

ORCID: 0000-0002-4013-3989 
easy to produce [10].

For high cesium adsorption capacity, both the amount of PB and the location of $\mathrm{PB}$ are important factors to be controlled. More PB may adsorb more cesium, but it may not work if PB is located inside an encapsulated supporting matrix. The mass transfer between the surrounding water and $\mathrm{PB}$ in composite materials is the key for producing an effective adsorbent. Therefore, the synthesis of PB on the surface of existing supporting materials, called in-situ synthesis, may be a better way to produce PB composite materials that have faster cesium adsorption. However, the surface attachment of $\mathrm{PB}$ may cause a leaching problem. To minimize PB leaching in water, strong binding between the $\mathrm{PB}$ and supporting materials is the key parameter to be investigated. Most research of PB immobilization have reported applying supporting materials that have an hydroxyl group [10], but ionic bonding between the hydroxyl group and PB particles is weak compared to other functional groups (carboxylic, amine) that can immobilize the particles [11]. To prevent the leakage of PB particles from supporting materials, the PB immobilization was applied from ligand $(-\mathrm{COOH})$ where it has been used in a semiconductor field [12, 13].

The last criteria for successful development of $\mathrm{PB}$ based nano-composite Cs adsorbent are ease to produce. Most of previous work focuses on developing new composite materials for Cs adsorption, such as chitin, alginate, magnetic metal oxides, etc. They are innovative and successful, but there are lack of study on its practical application and economic approach. In this study, we developed a protocol to functionalize commercially available supporting materials to immobilize PB and make it stable for contaminated water remediation. The poly vinyl alcohol (PVA) sponge was used as a supporting material, which can be easily obtained in the market for cleaning purposes. Its porous nature allows water to penetrate into the sponge and provides room for in-situ PB synthesis. Moreover, commercial filter materials have already proven for their mechanical strength and stability.

Acrylic acid (AA) was used for the surface functionalization of PVA sponge to convert the hydroxyl group into a carboxylic group for better PB attachment. Because AA includes double bond and carboxylic groups that have negative charge, AA is capable of trading electrons to alkali metals [14]. This approach overcomes the weak bonding of hydroxyl groups in previous studies and it can be applied to various supporting materials that contain hydroxyl groups. Thus, this study tried to graft an AA polymer to organic supporting material (PVA sponge) so it can endure in water treatment plants.

This work aimed to develop an approach to produce a PB based composite adsorbent for radioactive cesium uptake. This approach includes using commercial materials as a supporting matrix, functionalizing it by simple chemical reaction to obtain a carboxylic group, and synthesizing PB in-situ with prepared support.

\section{Materials and Methods}

\subsection{Materials}

A PVA sponge was obtained from Samkwang trading company (Daegu, Korea). AA (99\%), potassium persulfate (KPS, 99\%), and sodium hydroxide $(\mathrm{NaOH}, 93 \%)$ were purchased from Samchun Chemical Reagent Corporation Ltd. (Gyeonggi-do, Korea). Iron(III) chloride hexahydrate $\left(\mathrm{FeCl}_{3} \cdot 6 \mathrm{H}_{2} \mathrm{O}, 97 \%\right)$ and potassium ferrocyanide trihydrate $\left(\mathrm{K}_{4}\left[\mathrm{Fe}(\mathrm{CN})_{6}\right] \cdot 3 \mathrm{H}_{2} \mathrm{O}, 99 \%\right)$ as a precursor for synthesizing PB were purchased from Duksan Chemical Reagent Corporation Ltd. (Seoul, Korea). All of the chemical reagents and solvents were of extra pure grade. The $\mathrm{Cs}(\mathrm{I})$ standard solution $(1,000 \mathrm{mg} / \mathrm{L})$ as ICP-MS analytical grade was obtained from Kanto Chemical corporation Inc. (Chuo-cu, Japan). The deionized water was used to prepare all solutions.

\subsection{Preparation of PB-PAA-PVA Sponge}

\subsubsection{PVA surface functionalization by $A A$}

The overall scheme of preparation is presented in Fig. 1. A polymer solution was prepared with $60 \mathrm{mg}$ of potassium persulfate and $10 \mathrm{~mL}$ of AA solution dispersed into $50 \mathrm{~mL}$ of deionized water

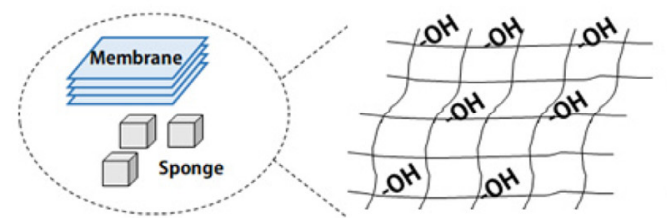

Polymer having hydroxyl group
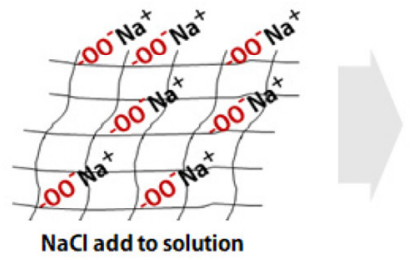

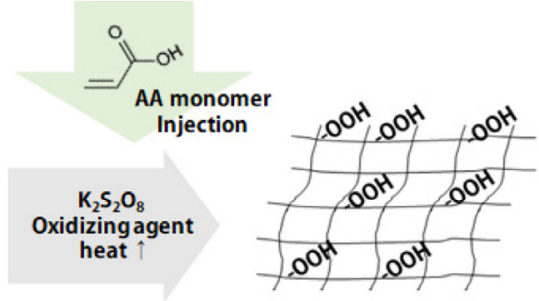

Grafting carboxylic group

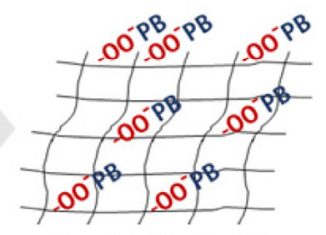

Prussian blue Growth

Fig. 1. Schematic of strategy synthesis for PB immobilization by AA modification. 
in a vessel. Afterwards, cubical PVA sponges that were $5 \times 5$ $\times 5 \mathrm{~mm}^{3}$ in size were immersed into the polymer solution. To remove dissolved oxygen from the vessel, the vacuum oven was filled with nitrogen gas and the reaction proceeded at $70^{\circ} \mathrm{C}$ for $6 \mathrm{~h}$. After the reaction ended, the PAA-PVA sponge was washed with a mixed solution, including a ratio 1:1 of ethanol and deionized water until the solution became transparent. Finally, the PAA-PVA sponge was dried at $60^{\circ} \mathrm{C}$.

\subsubsection{In-situ PB immobilization in PAA-PVA sponge}

In order to make the carboxylic group into an anion, a $250 \mathrm{mg}$ PAA-PVA sponge was immersed into $50 \mathrm{~mL}$ of $0.1 \mathrm{M} \mathrm{NaCl}$ solution. The modified/unmodified PVA sponge that was $5 \times 5 \times 5 \mathrm{~mm}^{3}$ in size was added into a $50 \mathrm{~mL}$ solution of $0.1 \mathrm{M} \mathrm{FeCl}_{3}$ for 24 $\mathrm{h}$ in order to attach $\mathrm{Fe}^{3+}$. Then, the PVA sponge was added into a $50 \mathrm{ml}$ solution of $0.1 \mathrm{M} \mathrm{K} \mathrm{Fe}_{4}(\mathrm{CN})_{6}$ for $5 \mathrm{~min}$ in order for the PB to synthesize. The obtained products were washed up to 5 times with $50 \mathrm{~mL}$ of deionized water. Afterwards, the obtained products were stored at $60^{\circ} \mathrm{C}$ and named as PB-PVA and PB-PAA-PVA, respectively.

\subsection{Optimization of Surface Modification}

The AA injection ratio for surface functionalization was optimized by measuring the weight changes by functionalization and the $\mathrm{Fe}^{3+}$ adsorption test using $250 \mathrm{mg}$ of a modified/unmodified PVA sponge in $50 \mathrm{~mL}$ of $1,000 \mathrm{mg} / \mathrm{L}$ of $\mathrm{Fe}^{3+}$ aqueous solution. The $\mathrm{Fe}^{3+}$ concentration in the solution was analyzed by an ICP-optical emission spectrometer (ICP-OES, OPTIMA 8300, Perkin-Elmer, USA). The effect of functionalization was evaluated by monitoring the changes in the functional group on the surface of the modified PVA sponge and pristine PVA sponge by using the Fourier-transform infrared spectroscopy (FTIR,
TENSOR27, and Bruker, Germany) recorded in the 400-4,000 $\mathrm{cm}^{-1}$ region. A scanning electron microscope with an energy dispersed spectroscopy (SEM/EDS, JSM-6700 F, JEOL, and Japan) was used to visualize surface modifications as well as analyze the chemical composition qualitatively.

\subsection{Cesium Adsorption Experiments}

The adsorption test of cesium ions in an aqueous solution using the PB-PAA-PVA sponge was carried out in batch experiments. In a typical batch adsorption equilibrium experiment, $100 \mathrm{mg}$ of the modified/unmodified PB-PVA sponge came into contact with $50 \mathrm{~mL}$ of a $5 \mathrm{mg} / \mathrm{L} \mathrm{Cs}^{+}$aqueous solution in a conical tube. The $\mathrm{pH}$ of the cesium aqueous solution was not adjusted and the reaction continued to be shaken at $303 \mathrm{~K}$ for $24 \mathrm{~h}$ in an agitator. The cesium ion concentration in a supernatant was measured by an ICP-Mass spectrometer (ICP-MS, NexlON 350D, Perkin-Elmer, USA). The sorption isotherm study of the adsorbed cesium ion at an equilibrium $\left(\mathrm{q}_{\mathrm{e}}\right)$ was calculated by Langmuir isotherm and Freundlich isotherm equations [15, 16]. And, effect of initial $\mathrm{pH}$ was investigated using $10 \mathrm{mg} / \mathrm{L} \mathrm{Cs}$ standard solution at different initial $\mathrm{pH}$ of $4,6,8,10$. The $\mathrm{pH}$ control was done by adding $1 \mathrm{M} \mathrm{HCl}$ and $\mathrm{NaOH}$ solution.

\section{Results and Discussion}

\subsection{Optimization of AA Modification}

As the AA injection ratio increased, the mass change ratio increased up to $38 \%$ as the gray bar shows in Fig. 2(a), which means more poly acrylic acid (PAA) formed in the PVA pore structure. A high content of carboxylic group content in the PAA structure led to
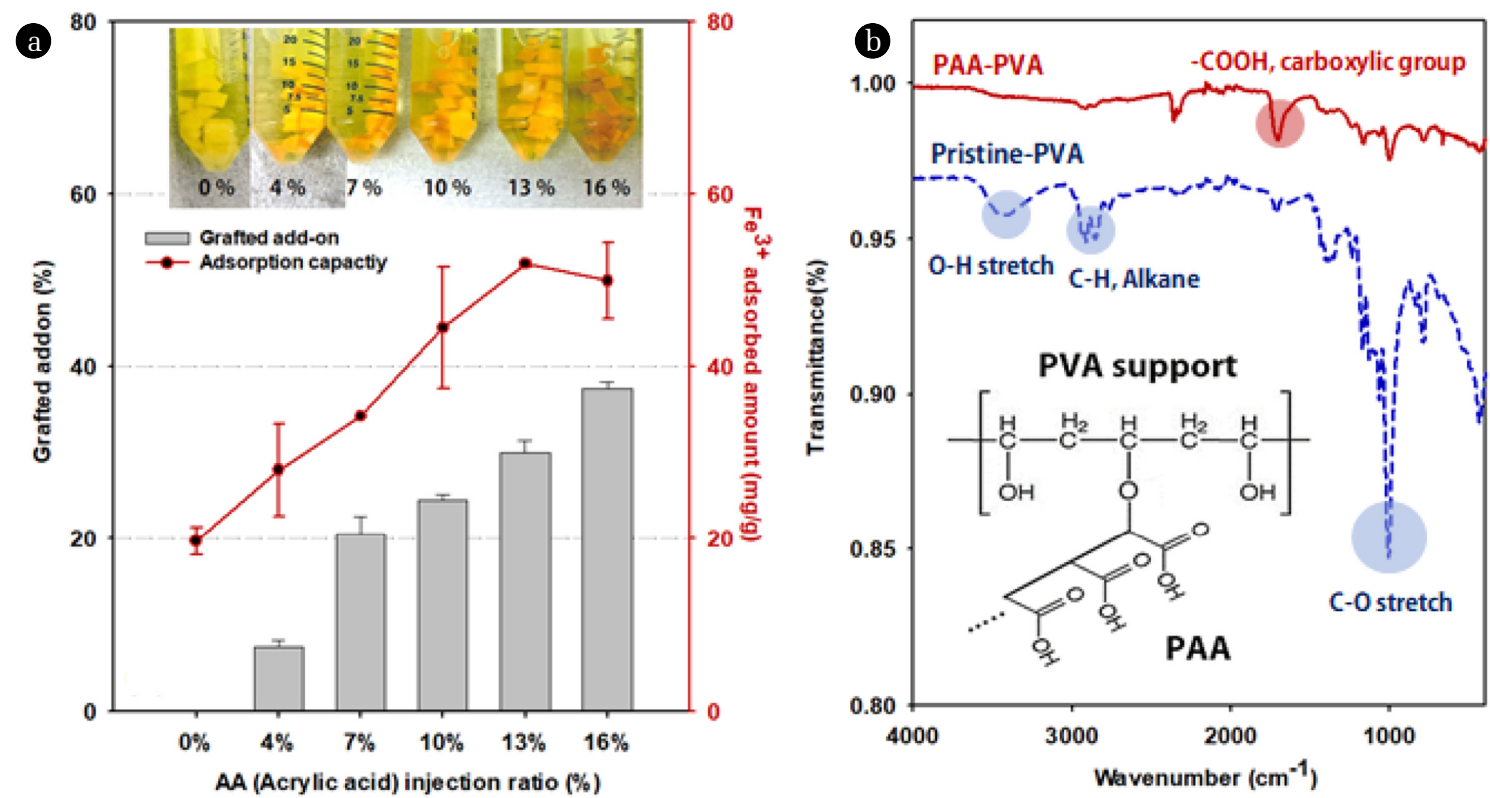

Fig. 2. (a) PVA sponge mass gain as a function of the $A A$ injection ratio and iron ion adsorption capacity of the modified PVA sponge in Fe ${ }^{3+}$ aqueous solution. (b) FTIR spectra of PVA and PVA modified by PAA. 
an increase in $\mathrm{Fe}^{3+}$ adsorption capacity. However, the $\mathrm{Fe}^{3+}$ adsorption capacity did not increase proportional to the addition of PAA. The maximum $\mathrm{Fe}^{3+}$ adsorption capacity was $52 \mathrm{mg} / \mathrm{g}$ at $13 \%$ of AA injection. It then decreased to $16 \%$ of AA injection. This was due to the pore blockage of the PVA sponge by the produced PAA network, which could be seen visually. Therefore, we decided to use $13 \%$ of the AA injection as the optimal condition for further experiments. Based on the chemical composition of $\mathrm{PB}\left(\mathrm{C}_{18} \mathrm{Fe}_{7} \mathrm{~N}_{18}\right)$, $52 \mathrm{mg} \mathrm{Fe} / \mathrm{g}$ can be calculated as $115 \mathrm{mg} \mathrm{PB} / \mathrm{g}$. In this point of view, $11.5 \%$ of $\mathrm{PB}$ content could be theoretically formed.

A change in functional groups was observed as a result of FTIR spectra shown in Fig. 2(b). Alcohol groups in the PVA sponge appeared at a spectra of 3,640-3,610 $\mathrm{cm}^{-1}$ and 1,320-1,000 $\mathrm{cm}^{-1}$ and have a deep shape in the dash line. After PAA modification, hydroxyl groups shifted to a $\mathrm{C}=\mathrm{O}$ stretch from carboxyl groups, which are 1,760-1,690 $\mathrm{cm}^{-1}$, and hydroxyl groups became weak compared to Pure-PVA [17]. This is similar to previous research related to PAA modification $[18,19]$. Therefore, the mechanism of chelating iron ion from modified PVA support can be explained by induced functional groups (-COOH).

In order to observe morphology changes, SEM images with EDS results (atom of total as weight \%) are presented in Fig. 3. A pure PVA sponge indicated polymer properties showing a relatively huge pore and a shape like chain-linked, as shown in Fig. 3(a). After AA modification to the PVA sponge, PB-PAA-PVA was
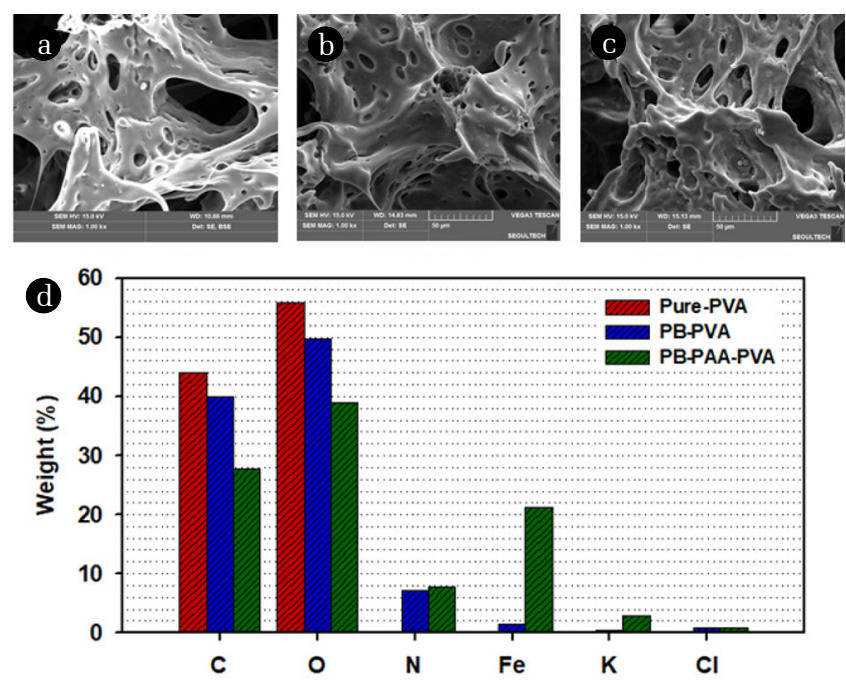

Fig. 3. SEM images $(\times 1,000$ magnification) of surface of (a) Pure-PVA, (b) PB-PVA, (c) PB-PAA-PVA, and (d) EDS data corresponded with each PB-PVA sponge.
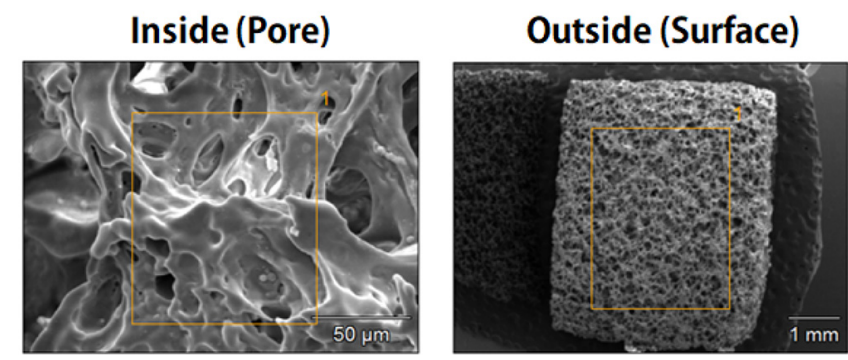

Fig. 4. SEM images of PB-PAA-PVA on inside (pore) and outside (surface).

photographed as having a reduced pore than pure PVA after changing its shape, as shown in Fig. 3(c). As a basis for supporting this, EDS data in Fig. 3(d) show that PB-PAA-PVA (green bar) contain more Fe atoms than Pure-PVA (red bar) and PB-PVA (blue bar). This means that the carboxylic group modified the PVA captured iron atom and it was a decisive influence of PB particles growing on their surface. However, nitrogen content did not show any significant difference. Nitrogen is another element to confirm successful PB formation. Therefore, it can be concluded that iron attachment was successful, but the converting to $\mathrm{PB}$ was not completed.

In order to confirm the homogeneity of PB formation, SEM/EDS analysis was performed for outer surface and cross-section of PB sponge (inside) as shown in Fig. 4. Atom distribution in the prepared adsorbent was not significantly different at inside pore and that surface, but portion of Fe was slightly higher in surface than that of inside, which indicates that penetration of precursor solutions were slightly inhibited by the overall structure of sponge.

\subsection{Advantage of PAA Modification on PB Immobilization}

One of the main objectives of this study was to prevent the further leaching of immobilized PB after it was disposed in a water environment. Fig. 5(a) shows the PB leaching of modified and unmodified PB immobilized PVA sponges during the washing step. More PB particles were leached to the solution in the case of the unmodified PVA sponge than the modified one. PB particles from the modified support, as shown on the left of Fig. 5(a), proved there was a strong attraction force, which was already verified as the carboxylic group on the FTIR spectra. They did not show the eluting of $\mathrm{PB}$ particles in an aqueous solution. The final products after washing are shown in Fig. 5(b). A darker blue color was observed in the modified one, thereby confirming the advantage of PAA modification.

Table 1. EDS Data Corresponded with Inside (Pore) and Outside (Surface) of PB-PAA-PVA Sponge

(Unit: wt\%)

\begin{tabular}{cccccccc}
\hline \multicolumn{1}{r}{ Atom } & C & N & O & K & Cl & Fe & Na \\
Type & 27.72 & 7.81 & 39 & 3.01 & 0.9 & 21.23 & 0.32 \\
PB-PAA-PVA (Inside) & 26.41 & 6.88 & 35.54 & 3.43 & 0.73 & 26.62 & 0.4 \\
\hline
\end{tabular}



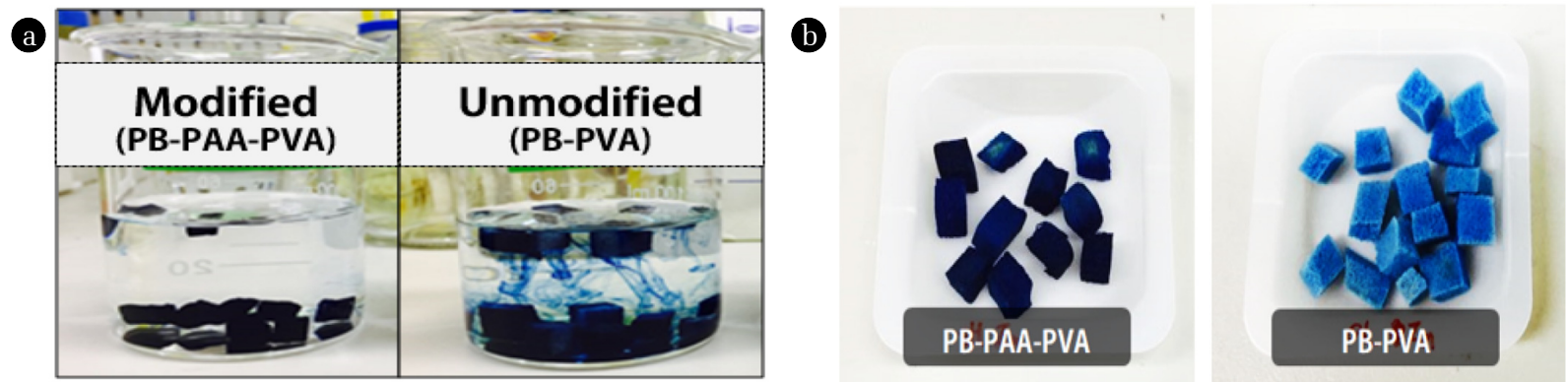

Fig. 5. (a) Image of eluting PB particles from the modified/unmodified PVA supports. (b) Final product after washing step.
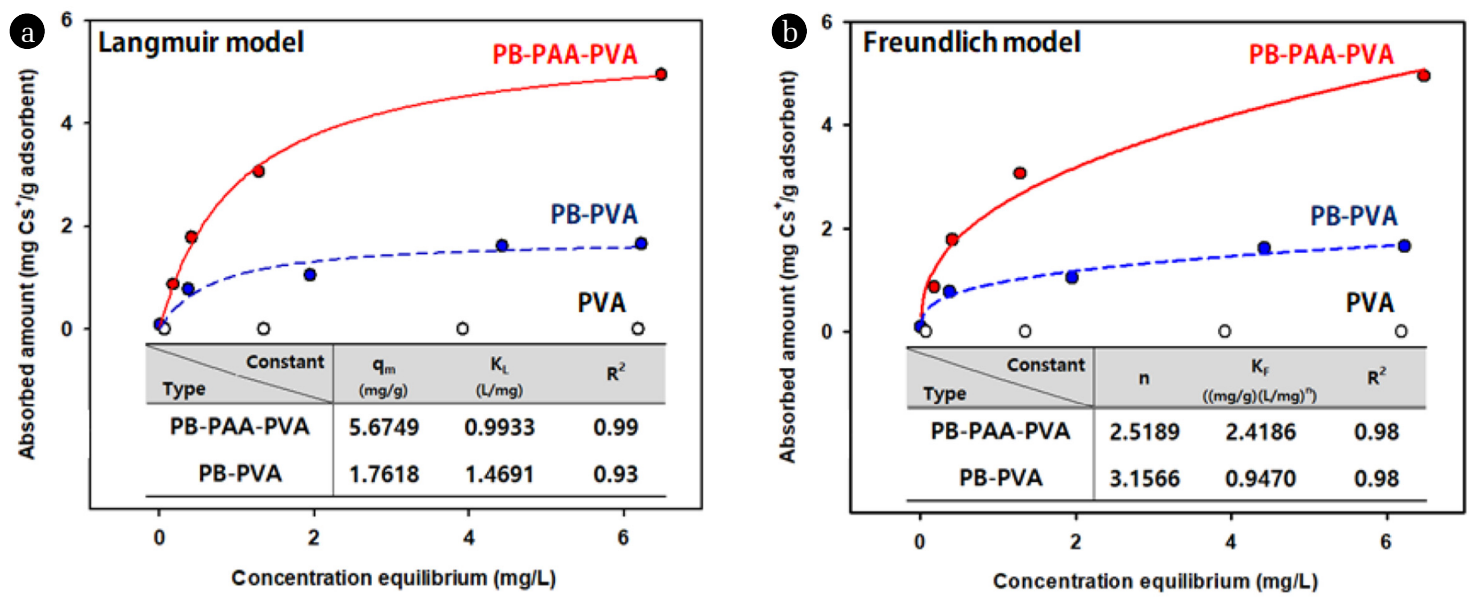

Fig. 6. Sorption isotherms curve fitted by (a) Langmuir \& (b) Freundlich models of the modified/unmodified PB-PVA sponge, respectively.

\subsection{Adsorption Study in $\mathrm{Cs}^{+}$Aqueous Solution}

The $\mathrm{Cs}^{+}$sorption isotherm was obtained for pure PVA, PB-PVA, and PB-PAA-PVA, as shown in Fig. 6. The pristine-PVA was not observed on adsorbing any $\mathrm{Cs}^{+}$, which clearly shows the effectiveness of PB. The maximum adsorption capacity of $\mathrm{Cs}(\mathrm{I})$ calculated by the Langmuir isotherm on PB-PAA-PVA calculated to 5.675 mg/g while PB-PVA only had $1.762 \mathrm{mg} / \mathrm{g}$ as its adsorption capacity. Such an increase of Cs(I) adsorption capacity was attributed by the increased amount of PB immobilized by PAA modification already revealed by EDS results. The AA modification formed - $\mathrm{COOH}$ inside of porous PVA sponge, which provides stronger affinity towards $\mathrm{Fe}^{3+}$, which is a precursor for forming $\mathrm{PB}$ immobilization. Therefore, higher Cs(I) uptake capacity on the AA modified PVA-PB adsorbent could be expected. The overall schematic diagram of cesium removal by PB-PAA-PVA was presented in Fig. 6.

The $n(n>1)$ constant of Freundlich model of two adsorbents apperared a favorable characteristic to adsorb cesium ion. Moreover, high vaule of $\mathrm{R}^{2}$ on both Langmur and Freundlich model were observed for PB-PAA-PVA, which indicate a behavior of adsorbing the cesium ions physically and chemically in pores [20]. The exclusive abilities of PB to adsorb hydrated $\mathrm{Cs}^{+}$are caused by regular lattice spaces surrounded by cyanide-bridged metals and proton-exchange mechanism acted on the specific $\mathrm{Cs}^{+}$adsorption [21, 22].

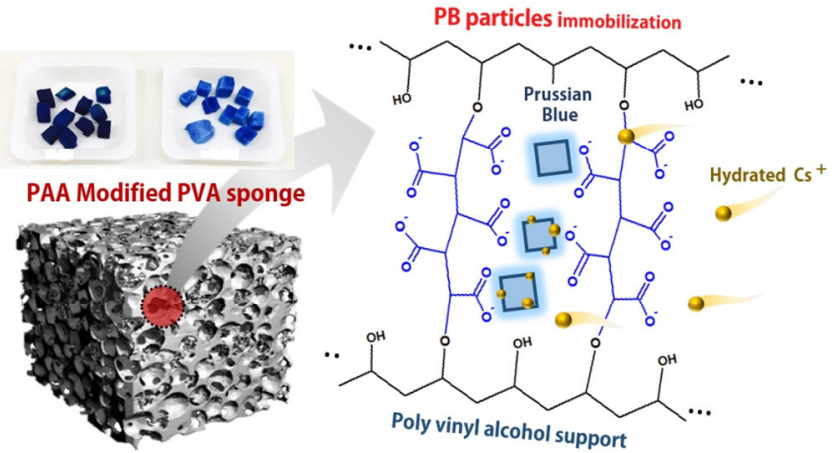

Fig. 7. Schematic diagram of PB particles immobilization on PAA modified PVA sponge.

The effect of $\mathrm{pH}$ on $\mathrm{Cs}^{+}$adsorption ability was also investigated. Overall, modified PB-PAA-PVA showed much higher adsorption capacity compared to unmodified PB-PVA. The unmodified adsorbent has maximum adsorption capacity lower than $2 \mathrm{mg} / \mathrm{g}$, while higher than $6 \mathrm{mg} / \mathrm{g}$ was obtained by modified one. The variation of $\mathrm{pH}$ was also significantly different with surface modification. In the case of modified PB-PAA-PVA, the final $\mathrm{pH}$ was maintained as around 6 and $\mathrm{pH}$ change was minimized. It was due to the effect of additional carboxylic acid groups supplied by AA modification, which acted as a buffering agent to uptake or leach $\mathrm{H}^{+}$ 

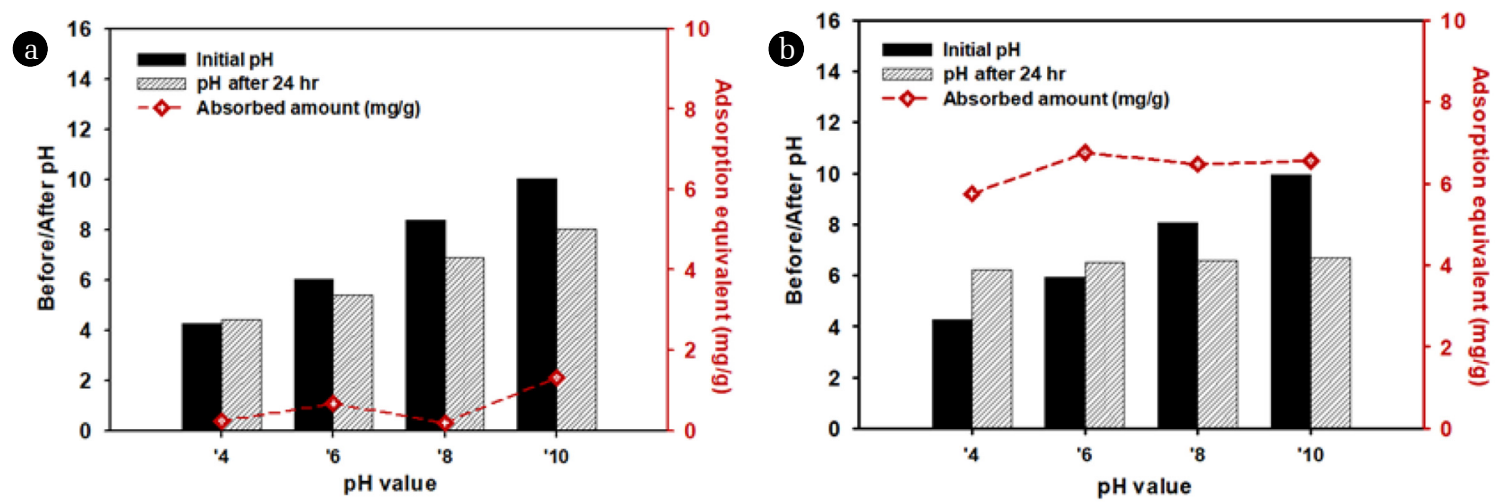

Fig. 8. The $\mathrm{pH}$ effects on $\mathrm{Cs}^{+}$adsorption ability of the unmodified PB-PVA (a) and modified PB-PAA-PVA (b) in $10 \mathrm{mg} / \mathrm{L}$ of $\mathrm{Cs}^{+}$solution during $24 \mathrm{~h}$.

ion. In this point of view, the developed adsorbent is able to be applied in various aqueous conditions having different $\mathrm{pH}$ without sacrificing its adsorption capacity.

\section{Conclusions}

In this study, we reported the simple strategy to prepare a $\mathrm{Cs}^{+}$ adsorbent using commercial polymeric material (PVA sponge) as a supporting matrix. The surface of PVA sponge was modified by PAA in order to provide better affinity toward PB. The PAA network was well prepared in the pore structure of the PVA sponge, but too much polymerization led to pore blockage, which inhibited mass transfer. The optimized AA injection ratio was determined to be $13 \%$. Surface functionalization by PAA grafting was identified by the FTIR spectra. The results of the FTIR spectra indicated that AA containing the carboxylic group successfully conjugated to the PVA sponge, as the alcohol group $\left(1,320-1,000 \mathrm{~cm}^{-1}\right)$ shifted to the carboxylic group $\left(1,760-1,690 \mathrm{~cm}^{-1}\right)$. The PAA network on the PVA sponge had a positive effect on attracting more PB as well as binding it more strongly, which was confirmed by the chemical composition obtained by SEM/EDS and the leaching test during washing. The maximum cesium adsorption capacity of PB-PAA-PVA increased to $5.675 \mathrm{mg} / \mathrm{g}$ compared to $1.762 \mathrm{mg} / \mathrm{g}$ for the PB-PVA. Simple modification using acrylic acid can be suggested as a versatile option to functionalize support containing the hydroxyl group in order to formulate PB based composite materials.

\section{Acknowledgments}

This study was supported by the Creative Convergence Research Project (CAP-15-07-KICT) of the National Research Council of Science and Technology (NST).

\section{References}

1. Avery SV. Fate of caesium in the environment: Distribution between the abiotic and biotic components of aquatic and terrestrial ecosystems. J. Environ. Radioact. 1996;30:139-171.

2. McLaughlin PD, Jones B, Maher MM. An update on radioactive release and exposures after the Fukushima Dai-ichi nuclear disaster. Brit. J. Radiol. 2012;85:1222-1225.

3. Lehto J, Haukka S, Harjula R, Blomberg M. Mechanism of caesium ion exchange on potassium cobalt hexacyanoferrates(II). J. Chem. Soc. Dalton Trans. 1990;1990:1007-1011.

4. Bustos E, Godinez LA. Modified surfaces with nano-structured composites of Prussian blue and dendrimers. New materials for advanced electrochemical applications. Int. J. Electrochem. Sci. 2011;6:1-36.

5. Vincent T, Taulemesse JM, Dauvergne A, Chanut T, Testa F, Guibal E. Thallium(I) sorption using Prussian blue immobilized in alginate capsules. Carbohydr. Polym. 2014;99:517-526.

6. Vipin AK, Fugetsu B, Sakata I, et al. Cellulose nanofiber backboned Prussian blue nanoparticles as powerful adsorbents for the selective elimination of radioactive cesium. Sci. Rep. 2016;6:37009.

7. Jang S-C, Hong S-B, Yang H-M, et al. Removal of radioactive cesium using Prussian blue magnetic nanoparticles. Nanomaterials 2014;4:894-901.

8. Chen L, Wang X, Zhang X, Zhang H. 3D porous and redox-active Prussian blue-in-graphene aerogels for highly efficient electrochemical detection of $\mathrm{H}_{2} \mathrm{O}_{2}$. J. Mater. Chem. 2012;22:22090-22096.

9. Jang S, Kang S, Haldorai Y, et al. Synergistically strengthened 3D micro-scavenger cage adsorbent for selective removal of radioactive cesium. Sci. Rep. 2016;6:38384.

10. Vincent T, Vincent C, Guibal E. Immobilization of metal hexacyanoferrate ion-exchangers for the synthesis of metal ion sorbents - A mini-review. Molecules 2015;20:20582-20613.

11. Yang J, Wang $X$, Zhu $M$, Liu $H$, Ma J. Investigation of PAA/PVDF-NZVI hybrids for metronidazole removal: Synthesis, characterization, and reactivity characteristics. J. Hazard. Mater. 2014;264:269-277.

12. Jiangxuan S, Mingjiong Z, Ran Y, et al. Interpenetrated gel polymer binder for high-performance silicon anodes in lithium-ion batteries. Adv. Funct. Mater. 2014;24:5904-5910.

13. Hong GR, Lee SS, Jo Y, et al. Extremely low-cost, scalable oxide semiconductors employing poly(acrylic acid)-decorated 
carbon nanotubes for thin-film transistor applications. ACS Appl. Mater. Interf. 2016;8:29858-29865.

14. Smuleac V, Bachas L, Bhattacharyya D. Aqueous - Phase synthesis of PAA in PVDF membrane pores for nanoparticle synthesis and dichlorobiphenyl degradation. J. Membrane Sci. 2010;346:310-317.

15. Foo KY, Hameed BH. Insights into the modeling of adsorption isotherm systems. Chem. Eng. J. 2010;156:2-10.

16. Hameed BH, Salman JM, Ahmad AL. Adsorption isotherm and kinetic modeling of 2,4-D pesticide on activated carbon derived from date stones. J. Hazard. Mater. 2009;163:121-126.

17. Kharazmi A, Faraji N, Mat Hussin R, Saion E, Mat Yunus WM, Behzad K. Structural, optical, opto-thermal and thermal properties of ZnS-PVA nanofluids synthesized through a radiolytic approach. Beilstein J. Nanotechnol. 2015;6:529-536.

18. Ge H, Wang J. Ear-like poly (acrylic acid)-activated carbon nanocomposite: A highly efficient adsorbent for removal of Cd(II) from aqueous solutions. Chemosphere 2017;169:443-449.

19. Sarkar S, Hazra C, Chatti M, Sudarsan V, Mahalingam V. Enhanced quantum efficiency for $\mathrm{Dy}^{3+}$ emissions in water dispersible $\mathrm{PbF}_{2}$ nanocrystals. RSC Adv. 2012;2:8269-8272.

20. Ishizaki M, Akiba S, Ohtani A, et al. Proton-exchange mechanism of specific $\mathrm{Cs}^{+}$adsorption via lattice defect sites of Prussian blue filled with coordination and crystallization water molecules. Dalton Trans. 2013;42:16049-16055.

21. Feng S, Li X, Ma F, et al. Prussian blue functionalized microcapsules for effective removal of cesium in a water environment. RSC Adv. 2016;6:34399-34410.

22. Lee I, Kim S, Rethinasabapathy M, et al. Porous 3D Prussian blue/cellulose aerogel as a decorporation agent for removal of ingested cesium from the gastrointestinal tract. Sci. Rep. 2018;8:4540. 